TRANSACTIONS OF THE

AMERICAN MATHEMATICAL SOCIETY

Volume 356, Number 5, Pages 1781-1797

S 0002-9947(04)03559-7

Article electronically published on January 6, 2004

\title{
A NONSTANDARD RIEMANN EXISTENCE THEOREM
}

\author{
RAHIM MOOSA
}

\begin{abstract}
We study elementary extensions of compact complex spaces and deduce that every complete type of dimension 1 is internal to projective space. This amounts to a nonstandard version of the Riemann Existence Theorem, and answers a question posed by Anand Pillay.
\end{abstract}

\section{INTRODUCTION}

A compact complex manifold can be viewed as a first order structure in the language where there is a predicate for each analytic subset of its cartesian powers. Recall that an analytic set is one that is given locally by the vanishing of holomorphic functions. The analytic sets form the closed sets of a noetherian topology that is coarser than the underlying complex topology, and which we refer to as the (analytic) Zariski topology. For example, in the special case of a projective variety every analytic set is given (globally) by the vanishing of homogeneous polynomials (Chow's Theorem). Hence the analytic Zariski topology coincides with the usual algebraic Zariski topology on a projective variety.

There is a nice model theory of compact complex manifolds. Zilber has shown, in 17], that the theory of a compact complex manifold, viewed as a structure in this language of analytic sets, admits quantifier elimination and is of finite Morley rank. While compactness is essential here, smoothness is not. We can work instead with compact complex varieties, where by a complex variety we will mean a (possibly nonsmooth) reduced and irreducible complex analytic space. In fact, it is convenient to deal with all compact complex varieties at once: consider the many-sorted structure $\mathcal{A}$ where there is a sort for each compact complex variety, and where the language, $\mathcal{L}$, consists of a predicate for each analytic subset of a cartesian product of sorts. Sort by sort then, $\operatorname{Th}(\mathcal{A})$ admits quantifier elimination and is of finite Morley rank. Moreover, $\operatorname{Th}(\mathcal{A})$ admits the elimination of imaginaries (a sketch appears in [13] and details in [10]). A survey of this model-theoretic approach can be found in [9].

The projective varieties, which can be viewed as living definably on the projective line, play a distinguished role among the sorts of $\mathcal{A}$. One form of the Riemann Existence Theorem states that every compact complex curve is biholomorphic to a projective curve. In [13] Anand Pillay asked whether this is also true in elementary extensions of $\mathcal{A}$. At the level of types, this would say that every complete type (over parameters) in $\operatorname{Th}(\mathcal{A})$ of dimension 1 is internal to the projective line. For types over the standard model $\mathcal{A}$ itself (or equivalently over the empty set),

Received by the editors July 17, 2002.

2000 Mathematics Subject Classification. Primary 03C60; Secondary 32J99.

This work was supported by the Natural Science and Engineering Research Council of Canada. 
this is the Riemann Existence Theorem. Our main result is that it remains true over arbitrary sets of parameters in any elementary extension of $\mathcal{A}$. This can be translated back into the geometric language as follows: Let $X$ and $S$ be compact complex varieties, and suppose $X \rightarrow S$ is a fibre space of curves, i.e., a holomorphic surjection whose fibres over a nonempty Zariski open subset of $S$ are irreducible of dimension 1. Then, after base change if necessary, there exist a family $Y \rightarrow S$ of projective curves and a meromorphic map $h: X \rightarrow Y$ over $S$ such that for all $s$ in a nonempty Zariski open subset of $S$, the induced map $h_{s}: X_{s} \rightarrow Y_{s}$ is a holomorphic bijection. The argument relies on a model-theoretic interpretation of a theorem of Campana's from [1, as well as an investigation of the relationship between Moishezon morphisms and internality to the projective line. We conclude with an application that characterises the infinite fields interpretable in $\operatorname{Th}(\mathcal{A})$.

Let us recall some notation. Suppose $X$ and $Y$ are compact complex varieties. By the dimension of $X$ we mean its dimension as a complex analytic space. A property is said to hold for general (respectively, 'general') points of $X$, if it holds in a nonempty Zariski open subset of $X$ (respectively, in a countable intersection of nonempty Zariski open subsets). For example, a holomorphic surjection from $X$ to $Y$ is called a fibre space if its general fibre is irreducible - that is, the fibres over some nonempty Zariski open subset of $Y$ are irreducible. A holomorphic map $f: X \rightarrow Y$ is a modification if there exist proper analytic subsets $A \subset X$ and $B \subset Y$ such that $f$ restricts to a biholomorphic map from $(X \backslash A)$ to $(Y \backslash B)$. By a meromorphic map from $X$ to $Y$ we will mean a multivalued map (written $g: X \rightarrow Y$ ) whose graph, $\Gamma(g) \subset X \times Y$, is an irreducible analytic set such that the first coordinate projection restricts to a modification $\Gamma(g) \rightarrow X$. Off a proper analytic set $A \subset X$, $g$ is a well-defined holomorphic map to $Y$. Moreover, $g: X \rightarrow Y$ is holomorphic (on all of $X$ ) if and only if $\Gamma(g) \rightarrow X$ is an isomorphism. A meromorphic map $g: X \rightarrow Y$ is surjective if the second coordinate projection restricts to a surjection $\Gamma(g) \rightarrow Y$; and it is bimeromorphic if $\Gamma(g) \rightarrow Y$ is also a modification. Note that every irreducible analytic subset of $X \times Y$ whose general fibre over $X$ is a singleton gives rise to a meromorphic map from $X$ to $Y$; and this map is bimeromorphic if and only if the general fibre over $Y$ is also a singleton. While many of the notions from complex analytic geometry will be treated in some detail, more comprehensive introductions to the theory of complex analytic spaces can be found in [7] (for a classical treatment) and [15] (for a modern treatment).

The results presented here constituted part of my $\mathrm{PhD}$ thesis [11] from the University of Illinois at Urbana-Champaign. I would like to thank Anand Pillay for his guidance and supervision during the writing of that thesis. I would also like to thank Thomas Scanlon, in discussions with whom the observation contained in Corollary 4.6 was obtained. Finally, I am grateful to the University of California at Berkeley and The Fields Institute in Toronto, where the final preparations for this article were made.

\section{Elementary extensions of COMPACT COMPleX VARIETIES}

In classical algebraic geometry, algebraically closed fields serve as the universal domains in which all the objects live, and where "generic" points of varieties can be found. There does not seem to be a direct analogue of this in complex analytic geometry. Passing to elementary extensions of a standard universe is characteristic of the model-theoretic approach, and in this case, it is one of the "new" techniques 
that model theory brings to complex analytic geometry. Our purpose in this section is mainly to describe how the model theory of elementary extensions relates to the geometry of $\mathcal{A}$, and to describe techniques of passing from one to the other.

Fix a cardinal $\kappa>|\mathcal{L}|$, and let $\mathcal{A}^{\prime}$ be a $\kappa$-saturated elementary extension of $\mathcal{A}$ of size $\kappa$ (which exists by stability). We treat $\mathcal{A}^{\prime}$ as a universal domain for $\operatorname{Th}(\mathcal{A})$. A definable set will always mean an $\mathcal{L}$-definable set in $\mathcal{A}^{\prime}$ with parameters from $\mathcal{A}^{\prime}$, and a type will always mean a complete type over a subset of $\mathcal{A}^{\prime}$ of size strictly less than $\kappa$. If $X$ is a compact complex variety (hence a sort of $\mathcal{A}$ ), and $G \subset X$ is a definable subset, then we sometimes use the notation $G(\mathcal{A})$ and $G\left(\mathcal{A}^{\prime}\right)$ to distinguish between $G$ and its interpretation in $\mathcal{A}^{\prime}$ - or, stated differently, between the $\mathcal{A}$-points and the $\mathcal{A}^{\prime}$-points of $G$.

Definition 2.1. Suppose $F$ is an irreducible Zariski closed set from $\mathcal{A}$. The generic type of $F$ over $\mathcal{A}$ is the type

$$
p(x)=\{x \in F, x \notin H: H \text { a proper Zariski closed subset of } F\} .
$$

A generic point of $F$ over $\mathcal{A}$ is a realisation of the generic type in $F\left(\mathcal{A}^{\prime}\right)$.

Remark 2.2. 1. The above definition is well-founded in the sense that the generic type of an irreducible Zariski closed set is complete and consistent (by quantifier elimination and noetherianity).

2. By saturation, every irreducible Zariski closed set has a generic point. Conversely, every tuple from $\mathcal{A}^{\prime}$ is a generic point of some irreducible Zariski closed set in $\mathcal{A}$ - namely, its locus.

3. If $P$ is a $\emptyset$-definable property of points in $F$, then $P$ holds for general $x \in F$ if and only if it holds for a generic point.

4. Types over $\mathcal{A}$ are in fact over $\emptyset$, as all points of $\mathcal{A}$ are named in $\mathcal{L}$.

The generic type over $\mathcal{A}$ of a compact complex variety is a bimeromorphic invariant in the following strong sense: if $X, Y$ are compact complex varieties, then $X$ and $Y$ are bimeromorphic if and only if for some generic points $a$ and $b$, of $X$ and $Y$ respectively, $a$ and $b$ are interdefinable over $\mathcal{A}$. This will follow from the following lemma by symmetry:

Lemma 2.3. Suppose $X$ and $Y$ are compact complex varieties, $a$ is generic in $X$ over $\mathcal{A}$, and $b$ is generic in $Y$ over $\mathcal{A}$. Then $b \in \operatorname{dcl}(a)$ if and only if there is a meromorphic surjection $h: X \rightarrow Y$ such that $h(a)=b$.

Proof. Note that a meromorphic map is definable at a generic point of its domain, and hence the notation $h(a)$ makes sense; we are interpreting the definable map $h$ in the elementary extension $\mathcal{A}^{\prime}$.

The right-to-left direction is immediate. Now assume $b \in \operatorname{dcl}(a)$. There is a partial definable map $f: X \rightarrow Y$ whose interpretation in $\mathcal{A}^{\prime}$ takes $a$ to $b$. Let $G \subseteq X \times Y$ be the graph of $f$. By quantifier elimination, $G=S_{1} \backslash T_{1} \cup \cdots \cup S_{\ell} \backslash T_{\ell}$, where $S_{1}, \ldots, S_{\ell}$ are distinct irreducible Zariski closed subsets of $X \times Y$, and each $T_{i}$ is a proper Zariski closed subset of $S_{i}$. As the fibre of $G\left(\mathcal{A}^{\prime}\right)$ over $a$ is the singleton $\{b\}$, this must also be the case for some $S_{i} \backslash T_{i}$, say $i=1$. Now, as $a$ is a generic point of $X$, it follows that for general $x \in X,\left(S_{1} \backslash T_{1}\right)_{x}=G_{x}$ is a singleton. But for general $x \in X,\left(S_{1} \backslash T_{1}\right)_{x}$ is a dense Zariski open set in $\left(S_{1}\right)_{x}$. Hence for general $x \in X,\left(S_{1}\right)_{x}=G_{x}$ is a singleton. That is, $S_{1}$ is the graph of a meromorphic function $h: X \rightarrow Y$ such that $h(a)=b$. As $b$ is a generic point of $Y$, $h$ is a meromorphic surjection. 
Every definable set in $\mathcal{A}^{\prime}$ is of the form

$$
G\left(\mathcal{A}^{\prime}\right)_{s}=\left\{y \in Y\left(\mathcal{A}^{\prime}\right):(s, y) \in G\left(\mathcal{A}^{\prime}\right)\right\},
$$

where $X$ and $Y$ are sorts of $\mathcal{A}, G \subset X \times Y$ is definable over $\mathcal{A}$, and $s \in X\left(\mathcal{A}^{\prime}\right)$. In particular, $G\left(\mathcal{A}^{\prime}\right)_{s}$ is a nonstandard Zariski closed set if $G$ can be chosen to be Zariski closed. By quantifier elimination, every definable set is a finite boolean combination of nonstandard Zariski closed sets.

There is a more canonical description of nonstandard Zariski closed sets. Suppose $\Gamma=G\left(\mathcal{A}^{\prime}\right)_{s}$ is as above. Let $S \subset X$ be the locus of $s$ - so that $s \in S\left(\mathcal{A}^{\prime}\right)$ is a generic point. Letting $H=G \cap(S \times Y)$, the restriction of $G$ to $S$, we have $\Gamma=H\left(\mathcal{A}^{\prime}\right)_{s}$. That is, we obtain $\Gamma$ as a generic fibre over $S$ of the family of Zariski closed sets

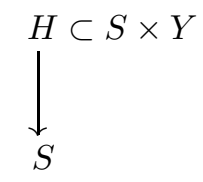

where $H \rightarrow S$ is the surjection induced by the first coordinate projection. Such a description is stable in the following sense: if $\Gamma$ is also given as a generic fibre of some other family $K \subset S \times Y$, then $K$ and $H$ have the same general fibres. That is, there is a nonempty Zariski open subset $U \subset S$ such that for every $u \in U, K_{u}=H_{u}$. It follows that $K$ and $H$ share those irreducible components that project onto $S$. The presentation of $\Gamma$ as a generic fibre of a Zariski closed subset of $S \times Y$ over $S$ is therefore unique up to irreducible components whose projections are proper subsets of $S$.

The above description also gives us a canonical way of going from one set of parameters to another. Suppose that $t$ is another parameter from $\mathcal{A}^{\prime}$. Then $\Gamma$ can be viewed as being definable over $(s, t)$ as well; letting $T$ be the locus of $(s, t), \Gamma$ can be presented as a generic fibre of some Zariski closed subset of $T \times Y$ over $T$. How do these two descriptions of $\Gamma$ compare? Let $\pi$ be the coordinate projection map taking $(s, t)$ to $s$. Transferring back to the standard model, we get a surjective holomorphic map $\pi: T \rightarrow S$. We can lift $H \rightarrow S$ to $T$ by base change:

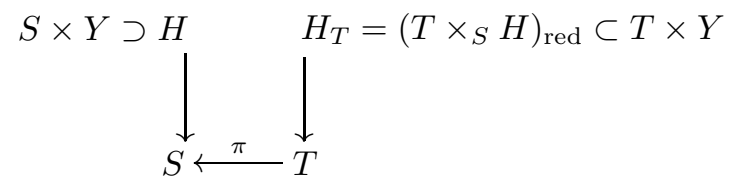

where $H_{T}=\{(v, y) \in T \times Y:(\pi(v), y) \in H\}$. Note that for any $v \in T$, the fibre of $H_{T}$ above $v$ is $H_{\pi(v)}$. That is, the fibres of $H_{T} \rightarrow T$ and $H \rightarrow S$ are the same, identified by $\pi$ on the base. In particular, $\Gamma$, being $\left(H_{T}\right)_{(s, t)}$, is a generic fibre of $H_{T} \rightarrow T$. The point here is that working with additional parameters in the universal domain corresponds to base change in the standard model.

Remark 2.4. Even if $H$ is irreducible, $H_{T}$ need not be. However, if the general fibres of $H \rightarrow S$ are irreducible (that is, $H \rightarrow S$ is a fibre space), then there is a unique irreducible component of $H_{T}$ that projects onto $T$. Indeed, let $Z_{1}, \ldots, Z_{\ell}$ be the irreducible components of $H_{T}$ that project onto $T$, and let $V \subseteq T$ be a nonempty Zariski open set over which the fibres of $H_{T}$ are irreducible. Shrinking $V$ further if necessary, we may assume that $Z_{1}, \ldots, Z_{\ell}$ are the only irreducible components of $H_{T}$ that have nonempty fibres over $V$. So for each $v \in V,\left(H_{T}\right)_{v}=\left(Z_{i}\right)_{v}$ for 
some $i \in\{1, \ldots, \ell\}$. This is a definable property, and hence, for $t \in T\left(\mathcal{A}^{\prime}\right)$ generic, $H_{T}\left(\mathcal{A}^{\prime}\right)_{t}=Z_{i}\left(\mathcal{A}^{\prime}\right)_{t}$ for some $i$, say $i=1$. Translating back into the standard model, we have that, for general $v \in T,\left(H_{T}\right)_{v}=\left(Z_{1}\right)_{v}$ and $\left(Z_{j}\right)_{v} \subseteq\left(Z_{1}\right)_{v}$ for all $2 \leq j \leq \ell$. But then $Z_{j} \subseteq Z_{1}$ for all $2 \leq j \leq \ell$, which is impossible unless $\ell=1$. That is, there is a unique irreducible component of $H_{T}$ that projects onto $T$, which we denote by $H_{(T)}$. Moreover, the general fibres of $H_{(T)} \rightarrow T$ agree with the general fibres of $H_{T} \rightarrow T$, and hence with those of $H \rightarrow S$. In particular, $H_{(T)} \rightarrow T$ is again a fibre space. We call $H_{(T)} \rightarrow T$ the strict pullback of $H \rightarrow S$ to $T$.

Using this description of nonstandard Zariski closed sets, we prove the following observation from Zilber [17:

Proposition 2.5. For any compact complex variety $X$, the nonstandard Zariski closed subsets of $X\left(\mathcal{A}^{\prime}\right)$ form the closed sets of a noetherian topology on $X\left(\mathcal{A}^{\prime}\right)$.

Proof. Suppose $X$ is a compact complex variety and $\Gamma_{1}, \Gamma_{2} \subset X\left(\mathcal{A}^{\prime}\right)$ are nonstandard Zariski closed sets obtained as generic fibres of $G_{1} \rightarrow S_{1}$ and $G_{2} \rightarrow S_{2}$ respectively. Let $S=S_{1} \times S_{2}$. Then $\Gamma_{1} \cap \Gamma_{2}$ (respectively $\Gamma_{1} \cup \Gamma_{2}$ ) is a generic fibre of $G_{1 S} \cap G_{2 S} \rightarrow S$ (respectively $G_{1 S} \cup G_{2 S} \rightarrow S$ ). The nonstandard Zariski closed sets are closed under finite unions and finite intersections.

Now suppose that the descending chain condition fails. That is, for some compact complex variety $X$ there are infinitely many nonstandard Zariski closed subsets $\Gamma_{i} \subset X\left(\mathcal{A}^{\prime}\right), i \in \omega$, where each $\Gamma_{i+1}$ is a proper subset of $\Gamma_{i}$. For each $i$, let $\Gamma_{i}$ be obtained as the generic fibre of $G_{i} \rightarrow S_{i}$. By base change, we may assume that there are surjective holomorphic maps $S_{i+1} \rightarrow S_{i}$. Since $\Gamma_{i+1} \subset \Gamma_{i}$, the general fibres of $G_{i+1} \rightarrow S_{i+1}$ are proper subsets of the general fibres of $G_{i} \rightarrow S_{i}$. This then yields an infinite properly descending chain of Zariski closed subsets in the 'general' fibre of $G_{0} \rightarrow S_{0}$ (i.e. in the fibres above a countable intersection of nonempty Zariski open subsets of $S_{0}$ ) - contradicting the descending chain condition of the Zariski topology on $X$.

A consequence of the descending chain condition is that every nonstandard Zariski closed set has a unique irredundant expression as the union of finitely many absolutely irreducible Zariski closed sets. We use the term absolutely irreducible in order to distinguish it from the following relative notion:

Definition 2.6. Suppose $B$ is a set of parameters from $\mathcal{A}^{\prime}$, and $\Gamma$ is a nonstandard Zariski closed set. We say that $\Gamma$ is over $B$ if it is definable with parameters from the set $B$. If $\Gamma$ is over $B$, then we say it is irreducible over $B$ if it cannot be written as the union of two proper nonstandard Zariski closed sets over $B$. Finally, we say that $\Gamma$ is absolutely irreducible if it is irreducible over any set of parameters $C \supset B$.

Note that $\Gamma$ is irreducible over $B$ if and only if it is irreducible over every finite tuple from $B$ over which it is defined. Moreover, if $\Gamma=G\left(\mathcal{A}^{\prime}\right)_{s}$, where $G \rightarrow S$ is a surjection of Zariski closed sets and $s \in S\left(\mathcal{A}^{\prime}\right)$ is generic, then $\Gamma$ is irreducible over $s$ if and only if $G$ has a unique irreducible component projecting onto $S$. For absolutely irreducible nonstandard Zariski closed sets, we have the following characterisation in terms of the standard model:

Lemma 2.7. Suppose $\Gamma=G\left(\mathcal{A}^{\prime}\right)_{s}$, where $\pi: G \rightarrow S$ is a surjection of irreducible Zariski closed sets and $s \in S\left(\mathcal{A}^{\prime}\right)$ is generic over $\mathcal{A}$. Then $\Gamma$ is absolutely irreducible if and only if $\pi$ is a fibre space. 
Proof. If $\pi: G \rightarrow S$ is a fibre space, then for any surjection $T \rightarrow S, G_{T}$ has a unique irreducible component $G_{(T)}$ that projects onto $T$ (see Remark 2.4). It follows that $\Gamma$ is irreducible over any tuple extending $s$, and hence is absolutely irreducible.

For the converse, assume that $\Gamma$ is absolutely irreducible. Let $f: G^{*} \rightarrow G$ be a resolution of $G$, and $\pi f: G^{*} \rightarrow S$ the composition. Now let

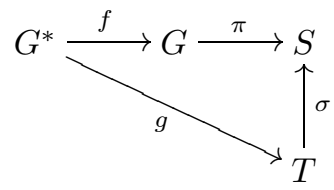

be the Stein factorisation of $\pi f$ (see $\S 1$ of [16]). In particular, $\sigma$ is finite-to-one and the fibres of $g$ are connected. But as $G^{*}$ is smooth, the general fibres of $g$ are also smooth (see 1.22 in [12]), and hence irreducible.

Now work in $\mathcal{A}^{\prime}$. As $\Gamma$ is absolutely irreducible, the above diagram implies that for some $t \in \sigma^{-1}(s), f$ maps $G^{*}\left(\mathcal{A}^{\prime}\right)_{t}$ surjectively onto $\Gamma=G\left(\mathcal{A}^{\prime}\right)_{s}$. This is a definable property of $s$, and hence in the standard model, for general $s^{\prime} \in S$, $G_{s^{\prime}}=f\left(G_{t^{\prime}}^{*}\right)$ for some $t^{\prime} \in \sigma^{-1}\left(s^{\prime}\right)$. As the general fibres of $g$ are irreducible, the general fibres of $\pi$ are irreducible, as desired.

Suppose $\Gamma$ is a nonstandard Zariski closed set over $B$. Then its absolutely irreducible components are defined over $\operatorname{acl}(B)$. Indeed, since there are only finitely many such components, and automorphisms of $\mathcal{A}^{\prime}$ that fix $B$ pointwise must permute them, each absolutely irreducible component of $\Gamma$ has only finitely many $B$ conjugates. By elimination of imaginaries and saturation, they must be definable over $\operatorname{acl}(B)$. Hence over (model-theoretically) algebraically closed sets, irreducibility and absolute irreducibility agree.

From now on, by a nonstandard variety we will mean a nonstandard absolutely irreducible Zariski closed set.

Definition 2.8. Suppose $\Gamma$ is a nonstandard Zariski closed set obtained as a generic fibre of a holomorphic surjection $G \rightarrow S$. The dimension of $\Gamma$ is by definition the dimension of the general fibres of $G \rightarrow S$.

The well-foundedness of Definition 2.8 follows from the definability of dimension in $\mathcal{A}$. Indeed, by a theorem of Remmert (the "dimension formula") the dimension of the general fibres of each irreducible component of $G$ that projects onto $S$ is constant. A proof of this fact can be found in Chapter 3 of 3 . Hence the general fibres of $G \rightarrow S$ are also of constant dimension. Moreover, if $\Gamma$ is obtained as a generic fibre of another analytic family $H \rightarrow T$, then by base change to $S \times T$, we have seen that the general fibres of $G \rightarrow S$ and $H \rightarrow T$ agree. Hence the dimension of a nonstandard Zariski closed set is well-defined.

Remark 2.9. It follows from Lemma 2.7 that if $\Gamma$ is a nonstandard variety, and $\Sigma \subset \Gamma$ is a proper nonstandard Zariski closed subset, then $\operatorname{dim} \Sigma<\operatorname{dim} \Gamma$. Indeed, we obtain $\Gamma$ as a generic fibre of a fibre space $G \rightarrow S$, and $\Sigma$ as a generic fibre of a surjection $H \rightarrow S$. As $\Sigma$ is a proper subset of $\Gamma$, the general fibres of $H \rightarrow S$ are proper subsets of the general fibres of $G \rightarrow S$ (which are irreducible). Hence the dimension of the general fibre of $H \rightarrow S$ is strictly less than that of $G \rightarrow S$.

Definition 2.10. Suppose $\Gamma$ is an irreducible nonstandard Zariski closed set over $B$. The generic type of $\Gamma$ over $B$ is the type $p(x)=\{x \in \Gamma, x \notin \Sigma\}$, where $\Sigma$ varies 
among all proper nonstandard Zariski closed subsets of $\Gamma$ over $B$. A generic point of $\Gamma$ over $B$ is a realisation of the generic type of $\Gamma$ in $\mathcal{A}^{\prime}$.

Suppose $c$ is a tuple from $\mathcal{A}^{\prime}$. The $B$-locus of $c$ is the smallest nonstandard Zariski closed set over $B$ containing $c$. The dimension of $c$ over $B$ (or of $\operatorname{tp}(c / B)$ ) is the dimension of the $B$-locus of $c$.

Again, by quantifier elimination and noetherianity, the generic type of a nonstandard irreducible Zariski closed set over $B$ is complete and consistent. Moreover, $c$ is generic in $\Gamma$ over $B$ if and only if $\Gamma$ is the $B$-locus of $c$. Saturation ensures that every nonstandard irreducible Zariski closed set over $B$ has a generic point.

We describes the geometric content of stationarity for types:

Lemma 2.11. Suppose a is a finite tuple, $B$ is a set of parameters, and $\Gamma$ is the $B$-locus of $a$. Then $\Gamma$ is pure-dimensional in the sense that the absolutely irreducible components of $\Gamma$ all have the same dimension. Moreover, the number of components is equal to the number of distinct extensions of $\operatorname{tp}(a / B)$ to $\operatorname{acl}(B)$. In particular, $\operatorname{tp}(a / B)$ is stationary if and only if $\Gamma$ is absolutely irreducible.

Proof. Let $\Sigma_{1} \subseteq \Gamma$ be the acl(B)-locus of $a$, and $\Sigma_{1}, \ldots, \Sigma_{\ell}$ the conjugates of $\Sigma_{1}$ under automorphisms that fix $B$ pointwise. Then $\Sigma_{1}, \ldots, \Sigma_{\ell}$ are absolutely irreducible, each of the same dimension, and their union - which is over $B$ and contains $a-$ is $\Gamma$. Hence they are the absolutely irreducible components of $\Gamma$, which is thereby pure-dimensional.

Moreover, the generic types of $\Sigma_{1}, \ldots, \Sigma_{\ell}$ over acl $(B)$ are (distinct) extensions of $\operatorname{tp}(a / B)$ (as they are conjugates of $\operatorname{tp}(a / \operatorname{acl}(B))$ under $B$-automorphisms). Conversely, if $q \in S(\operatorname{acl}(B))$ extends $\operatorname{tp}(a / B)$ and $b \models q$, then the $B$-locus of $b$ is $\Gamma$. As we saw above, this implies that the $\operatorname{acl}(B)$-locus of $b$ is an absolutely irreducible component of $\Gamma$, and so one of the $\Sigma_{i}$ 's. Hence $\operatorname{tp}(a / B)$ has exactly $\ell$ distinct extensions to $\operatorname{acl}(B)$, as desired. Finally, by elimination of imaginaries, stationarity means exactly that $\operatorname{tp}(a / B)$ has a unique extension to $\operatorname{acl}(B)$.

We recover nonforking independence (in the sense of the stable $\operatorname{Th}(\mathcal{A})$ ):

Lemma 2.12. Suppose $a$ and $b$ are finite tuples and $B$ is a set of parameters. Then $a$ is independent of $b$ over $B$ if and only if $\operatorname{dim}(a / B)=\operatorname{dim}(a / B b)$.

Proof. As $a$ is independent of $\operatorname{acl}(B)$ over $B$, and $\operatorname{dim}(a / B)=\operatorname{dim}(a / \operatorname{acl}(B))$, we may assume that $B=\operatorname{acl}(B)$. Let $\Gamma$ be the $B$-locus of $a$ and $\Sigma \subset \Gamma$ the $(B b)$-locus of $a$. Suppose $\operatorname{dim}(a / B)=\operatorname{dim}(a / B b)$. As $\Gamma$ is absolutely irreducible, this implies that $\Sigma=\Gamma$. Hence, every automorphism of $\mathcal{A}^{\prime}$ that fixes $B$ pointwise fixes $\Sigma$. As $\operatorname{tp}(a / B b)$ is the generic type of $\Sigma$, this means that every automorphism fixing $B$ fixes the set of realisations of $\operatorname{tp}(a / B b)$ setwise. It follows that $\operatorname{tp}(a / B b)$ is a nonforking extension of $\operatorname{tp}(a / B)$, as desired.

For the converse, suppose $a$ is independent of $b$ over $B$. Let $c \in \Gamma$ be generic in $\Gamma$ over $B b$. In particular $c$ is generic over $B$, and $\operatorname{sotp}(c / B)=\operatorname{tp}(a / B)$. Moreover, as $\Gamma$ is both the $(B b)$-locus and $B$-locus of $c, \operatorname{dim}(c / B b)=\operatorname{dim}(c / B)$. By the argument in the previous paragraph, this implies that $\operatorname{tp}(c / B b)$ is a nonforking extension of $\operatorname{tp}(c / B)=\operatorname{tp}(a / B)$. By stationarity, $\operatorname{tp}(c / B b)=\operatorname{tp}(a / B b)$, and hence $\operatorname{dim}(a / B b)=\operatorname{dim}(a / B)$. 
We end this section with a uniform version of Lemma 2.3.

Lemma 2.13. Suppose $S$ is a compact complex variety, and $X \rightarrow S$ and $Y \rightarrow S$ are fibre spaces over $S$. Suppose $s \in S\left(\mathcal{A}^{\prime}\right)$ is generic, a $\in X\left(\mathcal{A}^{\prime}\right)_{s}$ is generic over $s$, and $b \in Y\left(\mathcal{A}^{\prime}\right)_{s}$ is generic over $s$. Then $b \in \operatorname{dcl}(a)$ if and only if there is a meromorphic map $h$,

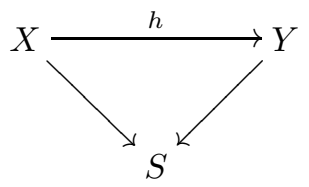

such that for general $s^{\prime} \in S, h_{s^{\prime}}: X_{s^{\prime}} \rightarrow Y_{s^{\prime}}$ is a meromorphic surjection; and $h_{s}(a)=b$.

Proof. The right-to-left direction is immediate. For the other direction, let $H$ be the locus of $(a, b)$ in $X \times Y$. Note that $H \subset X \times{ }_{S} Y$, as both $a$ and $b$ map to s. Moreover, $H\left(\mathcal{A}^{\prime}\right)_{a} \subset Y\left(\mathcal{A}^{\prime}\right)_{s}$ is the $a$-locus of $b$, and hence is a singleton by assumption. Since $a$ is generic in $X\left(\mathcal{A}^{\prime}\right)_{s}$ over $s$ and $b$ is generic in $Y\left(\mathcal{A}^{\prime}\right)_{s}$ over $s$, it follows that $H\left(\mathcal{A}^{\prime}\right)_{s} \subset X\left(\mathcal{A}^{\prime}\right)_{s} \times Y\left(\mathcal{A}^{\prime}\right)_{s}$ is one-to-one outside a proper $s$-definable nonstandard Zariski closed subset of $X\left(\mathcal{A}^{\prime}\right)_{s}$, and onto $Y\left(\mathcal{A}^{\prime}\right)_{s}$. As $s$ is generic in $S$ over $\mathcal{A}$, transferring back to the standard model we have that for general $s^{\prime} \in S$, $H_{s^{\prime}} \subset X_{s^{\prime}} \times Y_{s^{\prime}}$ is one-to-one over a nonempty Zariski open subset of $X_{s^{\prime}}$, and onto $Y_{s^{\prime}}$. This implies that $H_{s^{\prime}}$ is the graph of a surjective meromorphic map $X_{s^{\prime}} \rightarrow Y_{s^{\prime}}$. That is, $H$ induces a meromorphic map $h: X \rightarrow Y$ over $S$ such that for general $s^{\prime} \in S, h_{s^{\prime}}: X_{s^{\prime}} \rightarrow Y_{s^{\prime}}$ is a meromorphic surjection. By construction, $h_{s}(a)=b$.

Remark 2.14. Note that if $a$ and $b$ were interdefinable over $s$, then we would obtain a bimeromorphism $h: X \rightarrow Y$ over $S$.

\section{Projective And Moishezon morphisms}

In this section we discuss notions of relative algebraicity based on projective linear spaces and bundles. Our main purpose is to make explicit the relationship between these various notions, in a way that makes clear their model-theoretic content. While we intend to provide sufficient background and motivation, the reader is referred to Section 3 of 12 for a more detailed account.

Let $\mathbb{P}_{n}(\mathbb{C})$, or just $\mathbb{P}_{n}$, denote projective $n$-space viewed as a sort in $\mathcal{A}$. A projective variety is a compact complex variety which embeds in $\mathbb{P}_{n}$, for some $n \geq 0$. Note that the complex field, $(\mathbb{C},+, \times)$, is definable in $\mathbb{P}(\mathbb{C})$; here an identification of $\mathbb{C}$ with an affine open subset of $\mathbb{P}(\mathbb{C})$ is fixed once and for all. Conversely, every projective variety is naturally interpretable in $(\mathbb{C},+, \times)$; that is, an affine atlas and the associated transition functions are definable. Moreover, a fundamental fact (this is Chow's Theorem) is that every analytic set in projective space is algebraic; it is given by homogeneous polynomial equations. In this sense, algebraic geometry is purely embedded in $\mathcal{A}$; living definably on the sort $\mathbb{P}(\mathbb{C})$ and being the only structure induced on it by $\mathcal{A}$.

More generally, a Moishezon variety is a compact complex variety that is bimeromorphic to a projective variety. From the point of view of generic types, as witnessed by Lemma 2.3, Moishezon varieties are part of this "algebraic universe".

Fix a compact complex variety $S$ and consider the category of compact complex varieties over $S$. The analogue of projective space in this relative category is the 
notion of a projective linear space over $S$. Let $\mathcal{F}$ be a coherent analytic sheaf on $S$. We sketch a (local) construction of the projective linear space over $S$ associated to $\mathcal{F}$, denoted by $\pi: \mathbb{P}(\mathcal{F}) \rightarrow S$. Let $U \subset S$ be an open (in the complex topology) subset for which there exists a resolution of $\mathcal{F}_{U}$ as follows:

$$
\mathcal{O}_{U}^{p} \stackrel{\alpha}{\longrightarrow} \mathcal{O}_{U}^{q} \longrightarrow \mathcal{F}_{U} \longrightarrow 0
$$

where $\mathcal{O}$ denotes the structure sheaf of $S$, and $\mathcal{O}_{U}$ its restriction to $U$. As an $\mathcal{O}_{U^{-}}$ linear homomorphism, $\alpha$ can be represented by a $q \times p$ matrix $M=\left(m_{i j}\right)$, where each $m_{i j} \in \mathcal{O}_{U}$. Letting $X$ be coordinates for $U$ and $\left(Y_{1}: \cdots: Y_{q}\right)$ homogeneous coordinates for $\mathbb{P}_{q-1}$, we see that $\mathbb{P}(\mathcal{F})_{U}$ is the analytic subset of $U \times \mathbb{P}_{q-1}$ defined by the equations

$$
m_{1 i}(X) Y_{1}+\cdots+m_{q i}(X) Y_{q}=0
$$

for $i=1, \ldots, p$. One checks that $\mathbb{P}(\mathcal{F})_{U}$ depends only on the coherent sheaf $\mathcal{F}_{U}$, and not on the particular resolution chosen above. We then patch the $\mathbb{P}(\mathcal{F})_{U}$ to obtain $\mathbb{P}(\mathcal{F})$. The structure of a space over $S$ is induced on $\mathbb{P}(\mathcal{F})$ by the coordinate projection maps $U \times \mathbb{P}_{q-1} \rightarrow U$.

For each $s \in S$, the fibre $\mathbb{P}(\mathcal{F})_{s}$ is isomorphic to $\mathbb{P}_{r}$, where $r+1$ is the rank of $\mathcal{F}$ at $s$. In the special case when $\mathcal{F}$ is locally free, this rank is constant and $\pi: \mathbb{P}(\mathcal{F}) \rightarrow S$ is called a projective bundle over $S$. Projective bundles are locally trivial in the following strong sense. There are an open cover $\left\{U_{i}\right\}$ of $S$ and local trivialisations

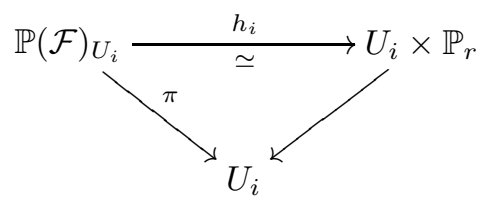

such that the induced transition functions

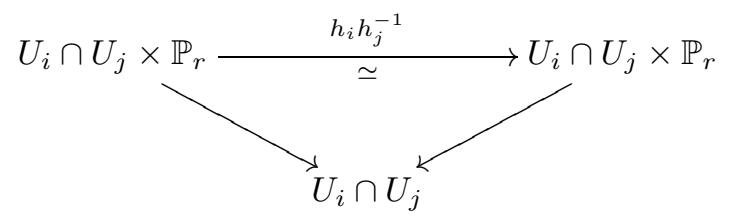

are of the form $h_{i} h_{j}^{-1}(x, p)=\left(x, g_{i j}(x)(p)\right)$, where $g_{i j}: U_{i} \cap U_{j} \rightarrow P G L(r, \mathbb{C})$ is holomorphic. In other words, a projective bundle over $S$ of rank $r$ is locally a product of the base with projective $r$-space, where the transition functions fix the base points while permuting the fibres as elements of the projective general linear group. A trivial projective bundle over $S$ is one of the form $S \times \mathbb{P}_{r} \rightarrow S$. This corresponds to the free analytic sheaf of rank $r+1$ on $S$.

A projective morphism $f: X \rightarrow S$ of compact complex varieties is then a holomorphic map that factors through an embedding into a projective linear space over $S$. That is, there are a coherent analytic sheaf $\mathcal{F}$ on $S$ and a holomorphic map $g: X \rightarrow \mathbb{P}(\mathcal{F})$,

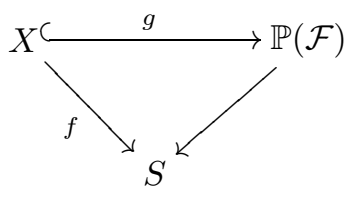


such that $g$ is an isomorphism with its image. In this case, we also say that $X$ is projective over $S$. The idea is as follows: if we take projective linear spaces over $S$ to be the relativisation of the notion of projective space, then projective morphisms are the natural analogue of projective varieties. Indeed, if $S$ is a point, then $\mathbb{P}(\mathcal{F})$ is a projective space and $X$ is a projective variety. Similarly, we obtain a relative notion of Moishezon: a Moishezon morphism is a holomorphic map $h: Y \rightarrow S$ that is bimeromorphic over $S$ to a projective morphism. That is, there is a projective morphism $f: X \rightarrow S$ and a meromorphic map $\sigma$,

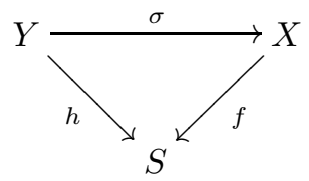

such that, for general $s \in S, \sigma_{s}: Y_{s} \rightarrow X_{s}$ is a bimeromorphism.

The following lemma says that if we are willing to pass to a bimeromorphically equivalent situation, as is justified from the point of view of generic types, then we may work with projective bundles rather than projective linear spaces.

Lemma 3.1. Suppose $f: X \rightarrow S$ is a projective morphism whose general fibres are irreducible. There are a modification $\sigma: T \rightarrow S$ and a projective bundle $\mathbb{P}(\mathcal{E}) \rightarrow T$ such that $X_{(T)}$ is isomorphic to a subvariety of $\mathbb{P}(\mathcal{E})$ over $T$ :

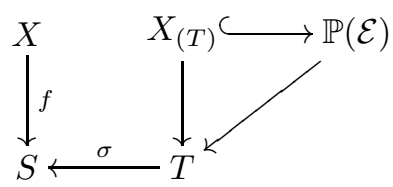

Proof. This is a known consequence of Hironaka's Flattening Theorem (see the proof of 5.1 in [5]). Suppose the projectivity of $f$ is witnessed by a coherent analytic sheaf $\mathcal{F}$ on $S$ such that $X$ is a subvariety of $\mathbb{P}(\mathcal{F})$ over $S$. By Hironaka's Flattening Theorem, there is a modification $\sigma: T \rightarrow S$ such that the quotient of $\sigma^{*}(\mathcal{F})$ by its torsion elements $\mathcal{E}$ is a flat, and hence locally-free, sheaf on $T$. The strict pullback of $X \rightarrow S$ to $T$ is a subvariety of the projective bundle $\mathbb{P}(\mathcal{E})$ over $T$.

Remark 3.2. Recall that a modification is, in particular, a bimeromorphism. Hence $X_{(T)} \rightarrow T$ is a bimeromorphic copy of the original $X \rightarrow S$.

The notion of a projective morphism may still seem somewhat weak. From the model-theoretic point of view, one might ask that a generic fibre of a projective morphism be a nonstandard Zariski closed set in projective space. That is, one might expect a projective morphism to factor through a trivial projective bundle over the base, $S \times \mathbb{P}_{n} \rightarrow S$. Lemma 3.1 says that this is the case locally (in the complex topology, after a bimeromorphic base change if necessary). It is not the case globally. The main point seems to be that we need to allow for additional parameters from $\mathcal{A}^{\prime}$. In the standard model this means allowing arbitrary (that is, not just bimeromorphic) base change: 
Lemma 3.3. If $\mathbb{P}(\mathcal{E}) \rightarrow S$ is a projective bundle, then for some $n \geq 0$, there are a holomorphic surjection $\pi: T \rightarrow S$ and a bimeromorphic map $g$,

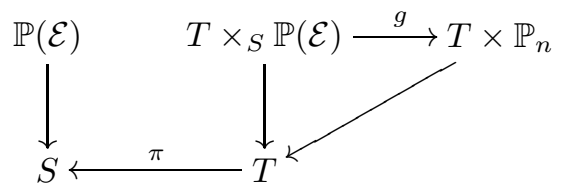

such that, for general $t \in T, g_{t}: \mathbb{P}(\mathcal{E})_{\pi(t)} \rightarrow \mathbb{P}_{n}$ is an isomorphism.

Proof. We could not find a reference for this fact in the literature, though it seems implicit in 5]. In any case, we use Fujiki's work on holomorphic fibre bundles [4] to give a proof.

We also use Douady spaces, a discussion of which can be found in [2. A survey of the theory of Douady spaces and their relevance to the model theory of compact complex varieties can also be found in [9]. Briefly, if $X$ is a complex analytic space, then the Douady space of $X$, denoted by $D(X)$, is a complex analytic space which parametrises the analytic subspaces of $X$. If $X$ is projective, $D(X)$ is the Hilbert scheme, and its components are themselves projective. In general, the components of the Douady space need not be compact. If $X \rightarrow S$ is a holomorphic surjection of complex analytic spaces, then the relative Douady space of $X$ over $S$, denoted by $D(X / S) \rightarrow S$, is an analytic space over $S$ whose fibres are the Douady spaces of the corresponding fibres of $X \rightarrow S$. If $X$ and $S$ are compact, and $X$ is projective over $S$, then the components of $D(X / S)$ are also compact and projective over $S$.

Let $\operatorname{rank}(\mathcal{E})=n+1$. For fixed $s \in S, \mathbb{P}(\mathcal{E})_{s}$ is isomorphic to $\mathbb{P}_{n}$. Any such isomorphism can be identified with its graph, which can in turn be identified with a point in the Douady space of $\mathbb{P}(\mathcal{E})_{s} \times \mathbb{P}_{n}$. The set of all such isomorphisms (for fixed $s$ ) forms a subset $U_{s}$ of $D\left(\mathbb{P}(\mathcal{E})_{s} \times \mathbb{P}_{n}\right)$. Varying $s$ in $S$, we obtain a subset, denoted by $\operatorname{Isom}_{S}\left(\mathbb{P}(\mathcal{E}), S \times \mathbb{P}_{n}\right)$, of the relative Douady space of $\mathbb{P}(\mathcal{E}) \times_{S}\left(S \times \mathbb{P}_{n}\right)$ over $S$. That is, there is an induced map $\operatorname{Isom}_{S}\left(\mathbb{P}(\mathcal{E}), S \times \mathbb{P}_{n}\right) \rightarrow S$ such that for all $s \in S, \operatorname{Isom}_{S}\left(\mathbb{P}(\mathcal{E}), S \times \mathbb{P}_{n}\right)_{s}=U_{s}$. Let $U=\operatorname{Isom}_{S}\left(\mathbb{P}(\mathcal{E}), S \times \mathbb{P}_{n}\right)$. Fujiki has shown that $U$ is a Zariski open subset of $D\left(\mathbb{P}(\mathcal{E}) \times_{S}\left(S \times \mathbb{P}_{n}\right) / S\right)$, which accordingly inherits the structure of a complex analytic space.

Moreover, since $\mathbb{P}(\mathcal{E}) \times_{S}\left(\mathbb{P}_{n} \times S\right) \rightarrow S$ is projective, the components of its relative Douady space are compact. This implies that $U$ has a natural compactification, $I=\operatorname{Isom}_{S}^{*}\left(\mathbb{P}(\mathcal{E}), S \times \mathbb{P}_{n}\right)$, all of whose irreducible components project onto $S$. Let $T$ be an irreducible component of $I$, and let $V$ be the Zariski open subset of $T$ obtained by intersecting with $U$. We argue that this $T$ works, namely that $T \times{ }_{S} \mathbb{P}(\mathcal{E})$ is bimeromorphic to $T \times \mathbb{P}_{n}$ over $T$.

We first point out that $V \times{ }_{S} \mathbb{P}(\mathcal{E})$ is isomorphic to $V \times \mathbb{P}_{n}$ over $V$. It is not hard to see, by the definition of Isom, that what is required is a holomorphic section to

$$
\operatorname{Isom}_{V}\left(V \times_{S} \mathbb{P}(\mathcal{E}), V \times \mathbb{P}_{n}\right) \longrightarrow V \text {. }
$$

But, by the functoriality of Isom (see 1.1(b) of [4]),

$$
\operatorname{Isom}_{V}\left(V \times_{S} \mathbb{P}(\mathcal{E}), V \times \mathbb{P}_{n}\right) \approx V \times_{S} \operatorname{Isom}_{S}\left(\mathbb{P}(\mathcal{E}), S \times \mathbb{P}_{n}\right)
$$

over $V$. The right hand side is just $V \times{ }_{S} U$, and so the diagonal map $d: V \rightarrow V \times{ }_{S} U$ yields the desired section.

We want the above isomorphism to extend to a bimeromorphism from $T \times{ }_{S} \mathbb{P}(\mathcal{E})$ to $T \times \mathbb{P}_{n}$ over $T$. For this it is sufficient (and necessary) that $d$ extend to a 
meromorphic section to

$$
\operatorname{Isom}_{T}^{*}\left(T \times{ }_{S} \mathbb{P}(\mathcal{E}), T \times \mathbb{P}_{n}\right) \longrightarrow T
$$

(see Remark 2 to Definition 1 of [4]). But, by the functoriality of Isom* this time (Remark 4 to Definition 1 of the same paper),

$$
\operatorname{Isom}_{T}^{*}\left(T \times_{S} \mathbb{P}(\mathcal{E}), T \times \mathbb{P}_{n}\right) \approx T \times_{S} \operatorname{Isom}_{S}^{*}\left(\mathbb{P}(\mathcal{E}), S \times \mathbb{P}_{n}\right)
$$

over $T$. The right hand side is just $T \times{ }_{S} I$. As $T$ is an irreducible component of $I$, the diagonal $T \rightarrow T \times{ }_{S} I$ yields the desired extension to $d$.

We obtain a bimeromorphism $g: T \times{ }_{S} \mathbb{P}(\mathcal{E}) \rightarrow T \times \mathbb{P}_{n}$ over $T$. As $g$ restricts to an isomorphism from $V \times_{S} \mathbb{P}(\mathcal{E})$ to $V \times \mathbb{P}_{n}$ over $V$, and as $V$ is a nonempty Zariski open set in $T$, it follows that for general $t \in T, g_{t}$ is an isomorphism.

\section{Nonstandard ALgebraicity}

Fix a saturated elementary extension $\mathcal{A}^{\prime}$ of $\mathcal{A}$, which, as before, we treat as a universal domain for $\operatorname{Th}(\mathcal{A})$. Note that if $\left(\mathbb{C}^{\prime},+, \times\right)$ denotes the interpretation of the complex field in $\mathcal{A}^{\prime}$, then $\mathbb{P}(\mathbb{C})\left(\mathcal{A}^{\prime}\right)$, the interpretation of $\mathbb{P}(\mathbb{C})$ in $\mathcal{A}^{\prime}$, is the projective line over the algebraically closed field $\mathbb{C}^{\prime}$. That is, $\mathbb{P}(\mathbb{C})\left(\mathcal{A}^{\prime}\right)=\mathbb{P}\left(\mathbb{C}^{\prime}\right)$. Hence by a nonstandard projective variety we will mean a projective variety over the field $\mathbb{C}^{\prime}$ (identified with its $\mathbb{C}^{\prime}$-points).

It may be useful at this point to recall some results of stability theory.

Remark 4.1. Suppose $T$ is a stable theory, $M \models T$, and $S$ is a sort in $M$. Then $S$ is stably embedded in $M$ - that is, every definable subset of $S^{n}$ is definable with parameters from $S$. Indeed, this is an instance of the definability of types in stable theories (see 6.7 .8 of $[8]$ ): if $\phi(x, y)$ is a formula (without parameters), where $x$ is a tuple of variables belonging to the sort $S$, and $b$ is from the sort of $y$, then there is a formula $\chi(x)$ with parameters from $S$ such that $\models \chi(a)$ if and only if $\phi(a, y) \in \operatorname{tp}(b / S)$. Hence, $\chi(x)$ defines the same set as $\phi(x, b)$ and is over $S$.

The above remark, together with Chow's Theorem mentioned earlier, implies that the full structure induced on $\mathbb{P}\left(\mathbb{C}^{\prime}\right)$ by $\mathcal{A}^{\prime}$ is interpretable in the algebraically closed field $\left(\mathbb{C}^{\prime},+, \times,\right)$ (which is itself definable in the sort $\mathbb{P}\left(\mathbb{C}^{\prime}\right)$ ). A number of things follow from this, including the fact that the structure induced on $\mathbb{P}\left(\mathbb{C}^{\prime}\right)$ has elimination of imaginaries (as this is the case for pure algebraically closed fields). Note that this does not follow directly from elimination of imaginaries for $\operatorname{Th}(\mathcal{A})$ - we are representing definable quotient spaces in the sort $\mathbb{P}\left(\mathbb{C}^{\prime}\right)$ by definable sets also in that sort.

Definition 4.2. Suppose $T$ is a stable theory, $M \models T$ is a sufficiently saturated model, $p(x) \in S(A)$ is a stationary type, and $S$ is a sort of $M$. Then we say that $p$ is internal to $S$ if there exist $B \supseteq A$, a realisation $a \models p(x)$ that is independent from $B$ over $A$, and finitely many elements $x_{1}, \ldots, x_{n} \in S$, such that $a \in \operatorname{dcl}\left(B, x_{1}, \ldots, x_{n}\right)$.

Remark 4.3. If in addition the structure induced on $S$ eliminates imaginaries, then one can conclude that $a$ is interdefinable over $B$ with a tuple from $S$. Indeed, let $c$ be a code for the definable set $X:=\left\{x \in S^{n}: g(x)=a\right\}$, where $g$ is the $B$-definable function witnessing that $a \in \operatorname{dcl}\left(B, x_{1}, \ldots, x_{n}\right)$. By elimination of imaginaries $c$ can be chosen to be a tuple from $S$, and $c$ is interdefinable with $a$ over $B$. 
Proposition 4.4. Suppose $X$ and $S$ are compact complex varieties and $f: X \rightarrow S$ is a fibre space. Then the following are equivalent:

(i) For generic $a \in X\left(\mathcal{A}^{\prime}\right), \operatorname{tp}(a / f(a))$ is internal to $\mathbb{P}\left(\mathbb{C}^{\prime}\right)$.

(ii) For some $n \geq 0$, there are a compact complex variety $T$ and a holomorphic surjection $T \rightarrow S$ such that $X_{(T)}$ is bimeromorphic to a subspace of $T \times \mathbb{P}_{n}$ over $T$.

(iii) There are a compact complex variety $T$ and a holomorphic surjection $T \rightarrow S$ such that $X_{(T)} \rightarrow T$ is Moishezon.

Proof. It follows from the above remarks that $(i)$ is equivalent to there being a tuple $t$ containing $f(a)$ such that

- $a$ is independent of $t$ over $f(a)$, and

- $a$ is interdefinable, over $t$, with a tuple from $\mathbb{P}\left(\mathbb{C}^{\prime}\right)$.

For $(i)$ implies $(i i)$, suppose that such a $t$ exists. Let $T$ be the locus of $t$. As $f(a)$ is a subtuple of $t$, and as $f(a)$ is generic in $S$ over $\mathcal{A}$, we obtain a holomorphic surjection $T \rightarrow S$. We claim that $a$ is generic in $X_{f(a)}=\left(X_{(T)}\right)_{t}$ over $t$. Indeed, as $a$ is generic in $X_{f(a)}$ over $f(a)$, and as $a$ is independent of $t$ over $f(a)$, we have that $\operatorname{dim}(a / t)=\operatorname{dim} X_{f(a)}$. By absolute irreducibility, the $t$-locus of $a$ is $X_{f(a)}$, as claimed. The interdefinability of $a$ with a tuple from $\mathbb{P}\left(\mathbb{C}^{\prime}\right)$ over $t$ thus means that for some $n \geq 0$ there is a meromorphic map $g$,

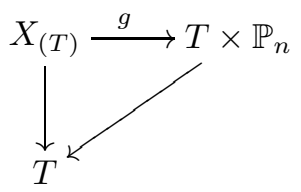

such that $g$ is bimeromorphic with its image over $T$.

Clearly (ii) implies (iii): the bimeromorphic image of $X_{(T)}$, being a subspace of the trivial projective bundle $T \times \mathbb{P}_{n}$, is projective. So by definition, $X_{(T)} \rightarrow T$ is Moishezon.

Finally, suppose (iii) holds. That is, there exist a compact complex variety $T$ and a holomorphic surjection $T \rightarrow S$ such that $X_{(T)} \rightarrow T$ is Moishezon. Applying Lemmas 3.1 and 3.3. and possibly base changing to a different $T$, we get that for some $n \geq 0$, there is a meromorphic map $h$,

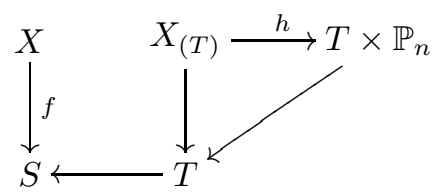

such that for general $t^{\prime} \in T, h_{t^{\prime}}$ is a meromorphic embedding of $\left(X_{(T)}\right)_{t^{\prime}}$ into $\mathbb{P}_{n}$. Now fix $a \in X\left(\mathcal{A}^{\prime}\right)$ generic, and choose $t \in T\left(\mathcal{A}^{\prime}\right)$ lying over $f(a)$ also generic over $\mathcal{A}$, and such that $a$ is independent of $t$ over $f(a)$. Then, as before, $a$ is generic in $X_{f(a)}$ over $t$, and $h_{t}$ witnesses that $a$ is interdefinable over $t$ with a tuple from $\mathbb{P}\left(\mathbb{C}^{\prime}\right)$. This proves that $\operatorname{tp}(a / f(a))$ is internal to $\mathbb{P}\left(\mathbb{C}^{\prime}\right)$, as desired.

Theorem 4.5. Suppose $p(x)$ is a stationary type of dimension 1 . Then $p(x)$ is internal to $\mathbb{P}\left(\mathbb{C}^{\prime}\right)$.

Proof. Let $p(x)=\operatorname{tp}(a / B)$ and let $\Gamma$ be the $B$-locus of $a$. As $p$ is stationary, Lemma 2.11 tells us that $\Gamma$ is absolutely irreducible. Let $s$ be a finite tuple from 
$B$ over which $\Gamma$ is defined. As the $s$-locus of $a$ is also $\Gamma$, it follows that $\operatorname{tp}(a / s)$ is stationary and $a$ is independent of $B$ over $s$ (by Lemma 2.12). It suffices to show that $\operatorname{tp}(a / s)$ is internal to $\mathbb{P}\left(\mathbb{C}^{\prime}\right)$.

Letting $X$ be the locus of $(s, a)$ and $S$ the locus of $s$, we have that $\Gamma=X\left(\mathcal{A}^{\prime}\right)_{s}$, and the general fibres of the induced holomorphic surjection $f: X \rightarrow S$ are irreducible and 1-dimensional. By Proposition 4.4 it is sufficient to show that after base change, $f: X \rightarrow S$ is Moishezon. Moreover, we may assume that $f$ admits a holomorphic section. Indeed, base changing $f: X \rightarrow S$ with itself, we obtain $X_{(X)} \rightarrow X$ which admits the diagonal section.

The theorem then follows from the following fact (see Lemma 3.10 in [2]): Any holomorphic surjection of compact complex varieties whose general fibres are irreducible and of dimension 1, and which admits a holomorphic section, is Moishezon. A proof of this fact can be found in Campana [1. (Théorème $2^{\prime}$ ). We briefly discuss the idea of the proof here. Assume that $X$ is smooth. Fixing general $s \in S$, we know by the Riemann Existence Theorem that $X_{s}$ is embeddable in projective space. Such an embedding is given by a very ample line bundle on $X_{s}$. Now if $\sigma: S \rightarrow X$ is a holomorphic section to $f$, then $\sigma(S)$ is a Cartier divisor that gives rise to a line bundle $\mathcal{E}$ on $X$. If $g$ is the genus of the general fibres of $f$, let $\mathcal{F}=\mathcal{E}^{\otimes 2 g+1}$. For general $s \in S, \mathcal{F}_{s}$ is a very ample line bundle on $X_{s}$. The main point is that the embeddings of the $X_{s}$ 's into projective space associated to the $\mathcal{F}_{s}$ 's are given uniformly by a meromorphic map

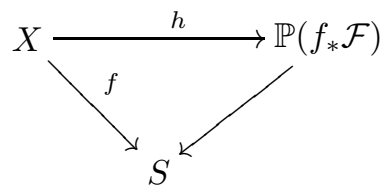

where $\mathbb{P}\left(f_{*} \mathcal{F}\right) \rightarrow S$ is the projective linear space associated to the coherent analytic sheaf $f_{*} \mathcal{F}$ on $S$ (of rank $g+2$ ). See page 19 of Ueno [16 for details on the construction of $h$. Since $h_{s}$ is an embedding for general $s \in S, h$ is a bimeromorphism onto its image. Hence $f: X \rightarrow S$ is Moishezon.

Let us make more explicit in what sense the above theorem can be considered as a nonstandard version of the Riemann Existence Theorem. From the equivalence of (i) and (ii) in Proposition 4.4, we see that if $\Gamma$ is a nonstandard variety whose generic type is internal to the projective sort, then $\Gamma$ is "generically definably isomorphic" to a nonstandard projective variety. That is, there are a projective variety $V$ over $\mathbb{C}^{\prime}$ and a definable isomorphism, using possibly additional parameters, between nonempty Zariski open subsets of $\Gamma$ and $V$. Considering the case of dimension 1, we can therefore conclude from Theorem 4.5 that up to finitely many points, every nonstandard curve is definably isomorphic to a nonstandard projective curve.

It is natural to ask for more (as was suggested to me by Dave Marker): namely, that every nonstandard curve is outright definably isomorphic to a projective curve (not just up to finitely many points). In fact, it is not hard to see, and was observed by myself and Thomas Scanlon, that this follows from the weaker statement

Corollary 4.6. Every nonstandard variety of dimension 1 is definably isomorphic to a nonstandard projective curve.

Proof. Suppose $\Gamma$ is a nonstandard curve. By Theorem 4.5 and Proposition 4.4 we obtain $\Gamma$ as a generic fibre of some fibre space of curves in the standard model 
$X \rightarrow S$ such that $X$ is bimeromorphic over $S$ to a subspace $V \subseteq S \times \mathbb{P}_{n}$. As $\mathbb{P}\left(\mathbb{C}^{\prime}\right)$ is stably embedded (see Remark 4.1), a generic fibre of $V$ over $S$ is definable with parameters in the sort $\mathbb{P}\left(\mathbb{C}^{\prime}\right)$. Hence, after taking a bimeromorphic copy over $S$, we may assume that $V \rightarrow S$ is obtained by base change from a fibre space of projective curves over a projective variety. We summarise this by the following diagram of compact complex varieties:

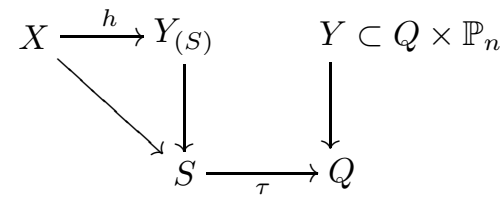

where

- $Q$ is projective and $Y$ is an algebraic family of projective curves over $Q$,

- $\tau: S \rightarrow Q$ is a holomorphic surjection,

- $h: X \rightarrow Y_{(S)}$ is a meromorphic map such that for general $s^{\prime} \in S, h_{s^{\prime}}$ is a bimeromorphism from $X_{s^{\prime}}$ to $\left(Y_{(S)}\right)_{s^{\prime}}=Y_{\tau\left(s^{\prime}\right)}$, and

- $\Gamma=X\left(\mathcal{A}^{\prime}\right)_{s}$ for some generic $s \in S\left(\mathcal{A}^{\prime}\right)$.

Replacing $Y$ with a resolution, we may also assume that the general fibres of $Y \rightarrow Q$ (and hence of $Y_{(S)} \rightarrow S$ ) are smooth curves. It follows that for general $s^{\prime} \in S$, $h_{s^{\prime}}^{-1}: Y_{\tau\left(s^{\prime}\right)} \rightarrow X_{s^{\prime}}$ is a holomorphic map (though $h_{s^{\prime}}$ may only be meromorphic). Note that while the parameters used to define $h_{s^{\prime}}^{-1}$ come from outside the projective sort, the definable equivalence relation on $Y_{\tau\left(s^{\prime}\right)}$ determined by $h_{s^{\prime}}^{-1}$ is over $\mathbb{P}(\mathbb{C})$ (again by stable embeddability).

On the other hand, by the Riemann Existence Theorem, $X_{s^{\prime}}$ is isomorphic to some projective curve $Z\left(s^{\prime}\right)$ living in some projective space $\mathbb{P}_{m}$ (where $m$ depends on $s^{\prime}$ ). Note that the isomorphism betweeen $X_{s^{\prime}}$ and $Z\left(s^{\prime}\right)$ does not necessarily vary uniformly with $s^{\prime}$. Nevertheless, composing with $h_{s^{\prime}}^{-1}$, we obtain a holomorphic map from $Y_{\tau\left(s^{\prime}\right)}$ to $Z\left(s^{\prime}\right)$ that produces the same equivalence relation on $Y_{\tau\left(s^{\prime}\right)}$ as $h_{s^{\prime}}^{-1}$. This is now occurring entirely on the sort $\mathbb{P}(\mathbb{C})$, where the induced structure is purely algebraic. By saturation in that sort (i.e., of the complex field) this happens at the generic fibres as well. That is, there are another algebraic family of projective curves $Z \rightarrow Q$ and a meromorphic map $g: Y \rightarrow Z$ over $Q$ such that if we lift $g$ to $Y_{(S)}$ by defining $g_{(S)}(s, y):=(s, g(y))$, then we obtain

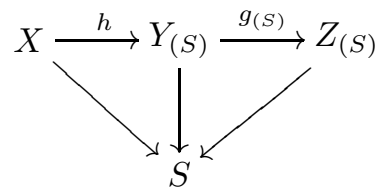

where $h_{s}^{-1}:\left(Y_{(S)}\right)_{s} \rightarrow \Gamma$ and $\left(g_{(S)}\right)_{s}:\left(Y_{(S)}\right)_{s} \rightarrow\left(Z_{(S)}\right)_{s}$ are defined everywhere and induce the same equivalence relation on $\left(Y_{(S)}\right)_{s}=Y_{\tau(s)}$. It follows that the relation obtained by composing (the graphs of) $h_{s}$ and $\left(g_{(S)}\right)_{s}$ determines a definable bijection between $\Gamma$ and the nonstandard projective curve $\left(Z_{(S)}\right)_{s}=Z_{\tau(s)}$.

Remark 4.7. The proof of Corollary 4.6] actually yields something more: the graph of the definable isomorphism that we construct is a (nonstandard) Zariski closed set (namely, the image of $\left(Y_{(S)}\right)_{s}$ in $X_{s} \times\left(Z_{(S)}\right)_{s}$ under $\left.h_{s}^{-1} \times\left(g_{(S)}\right)_{s}\right)$. Hence it comes from a family of graphs of holomorphic bijections in the standard model. This 
gives the geometric formulation mentioned in the introduction. Moreover, if $\Gamma$ were smooth in the sense that it came from a family of smooth curves in the standard model, then this graph would come from a family of graphs of isomorphisms in the standard model.

We conclude with an application of Theorem 4.5, suggested by Anand Pillay, that describes the infinite fields interpretable in $\operatorname{Th}(\mathcal{A})$. First of all, by elimination of imaginaries, it is sufficient to consider infinite fields definable in this theory. Working in the standard model, Pillay has shown that every such field in $\mathcal{A}$ is definably isomorphic to the complex field (see the discussion after 3.10 in [13]). The argument makes use of the Riemann Existence Theorem (as well as an observation of Kobi Peterzil). However, in order to understand infinite fields definable in $\operatorname{Th}(\mathcal{A})$, one must also consider elementary extensions of the standard model:

Corollary 4.8. Up to definable isomorphism, the only infinite field definable in $\mathcal{A}^{\prime}$ is $\left(\mathbb{C}^{\prime},+, \times\right)$.

Proof. Suppose $X$ is a compact complex variety and $F \subset X\left(\mathcal{A}^{\prime}\right)$ is an infinite definable set equipped with a definable field struicture $(F,+, \times)$. Let $\Gamma$ be the (absolute) Zariski closure of $F$. We first claim that $\operatorname{dim} \Gamma=1$. Indeed, let $s$ be a tuple from $\mathcal{A}^{\prime}$ over which $(F,+, \times)$ and $\Gamma$ are definable. Let $S$ be the locus of $s$. In the standard model, there is a definable subset $G \subset S \times X$ such that $G\left(\mathcal{A}^{\prime}\right)_{s}=F$. For general $s^{\prime} \in S, G_{s^{\prime}}$ is an infinite definable field in $\mathcal{A}$, and hence definably isomorphic to $\mathbb{C}$. In particular, the Zariski closure of $G_{S^{\prime}}$ is of dimension 1. It follows that the dimension of $\Gamma$ is 1 .

From Theorem 4.5 and the discussion following it, we obtain a definable embedding of $F$ into the projective sort. Carrying over the field structure to the image, we have a definable isomorphism of $(F,+, \times)$ with an infinitite field definable in the projective sort, $(H,+, \times)$. As all of the structure induced on $\mathbb{P}\left(\mathbb{C}^{\prime}\right)$ comes from the pure algebraically closed field $\left(\mathbb{C}^{\prime},+, \times\right)$, it follows that $(H,+, \times)$ is definable in $\left(\mathbb{C}^{\prime},+, \times\right)$. But the only infinite field definable in $\left(\mathbb{C}^{\prime},+, \times\right)$ is $\mathbb{C}^{\prime}$ itself, up to definable isomorphism (see [14], p.150). Hence, $(H,+, \times)$ is in turn definably isomorphic to $\left(\mathbb{C}^{\prime},+, \times\right)$.

\section{REFERENCES}

[1] F. Campana. Coréduction algébrique d'un espace analytique faiblement Kählérien compact. Inventiones Mathematicae, (63):187-223, 1981. MR 84e:32028

[2] F. Campana and T. Peternell. Cycle spaces. In Grauert et al. 6], pages 319-349. MR 96k:32001

[3] G. Fischer. Complex Analytic Geometry. Springer-Verlag, Berlin, 1976. MR 55:3291

[4] A. Fujiki. On a holomorphic fibre bundle with meromorphic structure. Publications of the Research Institute for Mathemtaical Sciences, 19(1):117-134, 1983. MR 84m:32038

[5] A. Fujiki. On the structure of compact complex manifolds in C. In Algebraic Varieties and Analytic Varieties, volume 1 of Advanced Studies in Pure Mathematics, pages 231-302. NorthHolland, Amsterdam, 1983. MR 85g:32045b

[6] H. Grauert, T. Peternell, and R. Remmert, editors. Several Complex Variables VII, volume 74 of Encyclopedia of Mathematical Sciences. Springer-Verlag, Berlin, 1994. MR 96k:32001

[7] R. Gunning and H. Rossi. Analytic functions of several complex variables. Prentice-Hall, Edgewood Cliffs, 1965. MR 31:4927

[8] W. Hodges. Model Theory. Cambridge University Press, Cambridge, 1993. MR 94e:03002

[9] R. Moosa. The model theory of compact complex spaces. To appear in the Proceedings of the Logic Colloquium '01 (Vienna).

[10] R. Moosa. On saturation and the model theory of compact Kähler manifolds. Preprint. 
[11] R. Moosa. Contributions to the model theory of fields and compact complex spaces. PhD thesis, University of Illinois, Urbana-Champaign, 2001.

[12] T. Peternell and R. Remmert. Differential calculus, holomorphic maps and linear structures on complex spaces. In Grauert et al. 6], pages 97-144. MR 96k:32001

[13] A. Pillay. Some model theory of compact complex spaces. In Workshop on Hilbert's tenth problem: relations with arithmetic and algebraic geometry, volume 270 of Contemporary Mathematics, Amer. Math. Soc., Providence, RI, 2000, pp. 323-338. MR 2001m:03076

[14] B. Poizat. Groupes stables. Nur al-Mantiq wal-Ma'rifah, Villeurbanne, 1987. MR 89b:03056

[15] R. Remmert. Local theory of complex spaces. In Grauert et al. [6], pages 10-96. MR 96k:32001

[16] K. Ueno. Classification Theory of Algebraic Varieties and Compact Complex Spaces, volume 439 of Lecture Notes in Mathematics. Springer-Verlag, Berlin, 1975. MR 58:22062

[17] B. Zilber. Model theory and algebraic geometry. In Proceedings of the 10th Easter Conference on Model Theory, Berlin, 1993.

The Fields Institute, 222 College Street, Toronto, Ontario, Canada M5T 3J1

Current address: Massachusetts Institute of Technology, Department of Mathematics, 77 Massachusetts Avenue, Cambridge, Massachusetts 02139-4307

E-mail address: moosa@math.mit.edu 